\title{
Assessment of Skin Toxicity in an in Vitro Reconstituted Human Epidermis Model Using Deep Learning
}

Fangyao Hu, ${ }^{*}$ Sara F. Santagostino, ${ }^{*}$ Dimitry M. Danilenko, ${ }^{\dagger}$ Min Tseng, ${ }^{*}$ Jochen Brumm,,${ }^{\ddagger}$ Philip Zehnder, ${ }^{*}$ and Kai Connie Wu*

From the Departments of Safety Assessment* and Nonclinical Biostatistics, ${ }^{\ddagger}$ Genentech, South San Francisco, California; and Danilenko Nonclinical Drug Development ${ }^{\dagger}$ Sedona, Arizona

Accepted for publication

December 28, 2021.

Address correspondence to Kai Connie Wu, Ph.D., or Fangyao Hu, Ph.D., Genentech, 1 DNA Way, South San Francisco, CA 94080. E-mail:wu.kai@gene. com or hu.fangyao@gene.com.

\begin{abstract}
Skin toxicity is a common safety concern associated with drugs that inhibit epidermal growth factor receptors as well as other targets involved in epidermal growth and differentiation. Recently, the use of a three-dimensional reconstructed human epidermis model enabled large-scale drug screening and showed potential for predicting skin toxicity. Although a decrease in epidermal thickness was often observed when the three-dimensional reconstructed tissues were exposed to drugs causing skin toxicity, the thickness evaluation of epidermal layers from a pathologist was subjective and not easily reproducible or scalable. In addition, the subtle differences in thickness among tissues, as well as the large number of samples tested, made cross-study comparison difficult when a manual evaluation strategy was used. The current study used deep learning and image-processing algorithms to measure the viable epidermal thickness from multiple studies and found that the measured thickness was not only significantly correlated with a pathologist's semi-quantitative evaluation but was also in close agreement with the quantitative measurement performed by pathologists. Moreover, a sensitivity of 0.8 and a specificity of 0.75 were achieved when predicting the toxicity of 18 compounds with clinical observations with these epidermal thickness algorithms. This approach is fully automated, reproducible, and highly scalable. It not only shows reasonable accuracy in predicting skin toxicity but also enables cross-study comparison and high-throughput compound screening. (Am J Pathol 2022, 192: 687-700; https://doi.org/10.1016/j.ajpath.2021.12.007)
\end{abstract}

The skin consists of two main layers: the epidermis, a thin stratified epithelium primarily composed of keratinocytes, and the dermis, a thick layer derived from mesenchymal cells. ${ }^{1}$ Any disruption of cell cycle or differentiation of the keratinocyte results in a disturbed skin barrier leading to changes in epidermal thickness and compromised function. Thus, a decrease in epidermal thickness is an important indicator for skin toxicities as well as for cutaneous disease. $^{2,3}$

The epidermal growth factor receptor (EGFR) regulates multiple functions in the keratinocyte, including proliferation, adhesion and migration, survival, and differentiation. EGFR mutants are therefore frequently associated with several cancers that include breast, lung, ovarian, cervical, bladder, esophageal, brain, and head and neck cancers. ${ }^{4-6}$ Given the EGFR expression in normal epithelial cells, therapies that target EGFR display on-target, off-tumor effects in the skin with evidence of rash, making skin toxicity a leading cause of dose reduction or treatment termination in patients undergoing cancer therapy, should the severity of rash be life-threatening. ${ }^{7}$ Similarly, maculopapular rash and/or acneiform eruptions of variable severity have been observed in patients treated with drugs targeting the mitogen-activated protein kinase and the phosphoinositide 3-kinase pathways, and closely mirror the cutaneous toxicity profile described for EGFR-targeting compounds in its onset, course, and treatment. ${ }^{8,9}$

\footnotetext{
Supported by Genentech, Inc.

Disclosures: F.H., S.F.S., M.T., J.B., P.Z., and K.C.W. are employees of Genentech, Inc., and are Roche stockholders. Danilenko Nonclinical Drug Development, LLC has no disclosures other than consulting for Genentech.
} 
There are no reliable in vivo models for predicting the potential of a systematically administered drug to induce skin toxicity before clinical trial. ${ }^{10}$ Fortunately, the last two decades have seen the advent of in vitro human skin models, which are valuable in qualitatively predicting clinical skin toxicity. ${ }^{11-13}$ These reconstituted human epidermal (RHE) models are metabolically and mitotically active threedimensional models of the normal human epidermis derived from cultured human epidermal keratinocytes. After culture with a drug of interest, pathologists can evaluate the RHE for signs of skin toxicity and use a semi-quantitative method to identify changes in epidermal thickness. ${ }^{14}$ However, this method is time-consuming and subjective, leading to longer drug development timelines and a hindered ability for cross-study comparisons of skin toxicity.

To combat the shortcomings of manually collected data, machine learning models designed to recognize and classify data are now used across disciplines, from cancer detection and monitoring to drug development and safety assessment. ${ }^{15}$ However, traditional machine learning algorithms cannot be trained on raw data but instead require timeintensive training on input data sets with well-defined data features. These features must be iteratively refined to ensure that the model has peak predictive power. ${ }^{16}$ Deep learning models based on neural networks were developed to overcome these limitations. Deep learning takes advantage of large data sets and general-purpose, nonlinear learning algorithms to train multiple hidden processing layers that are responsible for identifying different data features. The model itself defines these features based solely on the input data. ${ }^{15,16}$ In the last 5 years, deep learning methods have been applied to many classic drug development problems, including evaluating molecular structure activity relationships and patient outcome predictions. ${ }^{17,18}$

Deep learning models are especially well-suited for image analysis, segmentation, and classification, provided sufficiently large training sets of images with corresponding annotated data. ${ }^{16}$ Deep learning has been applied to many areas of medical imaging and drug toxicology. Other deep learning methods take advantage of toxicity-induced structural alterations in cells or tissues. ${ }^{19}$ This method has been used in vitro to assess toxicity based on changes in fluorescently-stained cell nuclei and to identify drug-induced structural changes in cardiomyocytes and hepatocytes that are too subtle for standard image analysis. ${ }^{20,21}$ Deep learning-based image analysis has also been applied to in vivo rodent models of ovarian toxicity as well as druginduced retinal atrophy and cardiomyopathy. ${ }^{22-24}$ Furthermore, a deep learning model was developed to predict radiation-induced toxicity in patients undergoing treatment for cancers of the hepatobiliary system. ${ }^{25}$ Finally, deep learning methods have been suggested as a means of identifying neurotoxicity using the robust segmentation framework that has already been developed for brain imaging. ${ }^{26}$ However, deep learning approaches have not yet been applied to preclinical skin toxicity analysis.
Herein, a deep learning segmentation model was used to identify the viable epidermis of the EpiDerm (MatTek Life Sciences, Ashland, MA), a human RHE model, in five skin toxicity studies evaluating drug compounds in clinical development. Samples were analyzed, and quantitative epidermal thickness end points were extracted. Four studies with manual pathologist evaluations were used to assess algorithm-extracted epidermal thickness. The algorithmextracted measurements were also compared with two studies with manual pathologist measurements. Finally, EpiDerm samples, each treated with compounds with either known or suspected clinical skin toxicity, were used to evaluate the utility of the method in identifying compounds at high risk for skin toxicity.

\section{Materials and Methods}

\section{Study Design}

Samples from five studies that evaluated the skin toxicity of various compounds were included in this retrospective study. Figure 1A summarizes the study design, which comprised three sections. A deep learning segmentation algorithm was used to segment the viable epidermal layer, and the viable epidermal thickness was computed from each sample in the three sections. In Section A, the viable epidermal thicknesses of the RHE samples in Studies 1 and 2 were scored by pathologists A and B, respectively, and Studies 4 and 5 were scored by pathologist C. Study 3 was not scored by pathologists. The scores were compared with the corresponding algorithm-measured thickness. In Section $\mathrm{B}$, pathologist $\mathrm{C}$ was provided image-viewing software to measure the viable epidermal thickness. The pathologistmeasured epidermal thickness was compared with the algorithm-measured thickness. In Section C, the segmented viable epidermal layers from all studies were quantified with an image-processing algorithm. The computed viable epidermal thickness for each compound from all studies was compared. Table 1 summarizes the five studies and the end points available for each study.

\section{Experiment Design and Protocols}

The three-dimensional in vitro human skin model, EpiDerm, was used for all studies. A total of 31 compounds with known or suspected clinical skin toxicity were evaluated for skin toxicity, some of which were evaluated in more than one study. Upon receipt of the RHE samples, each tissue culture insert was transferred to a well of a six-well plate containing $1 \mathrm{~mL}$ per well of prewarmed maintenance media and kept dry on top to maintain an air-liquid interface. The RHE samples were equilibrated at $37^{\circ} \mathrm{C}, 5 \%$ carbon dioxide overnight, and fed with fresh media the next day. One day later, the RHE samples were subjected to 4-day daily basolateral treatment with test articles at various concentrations or vehicle control dimethyl sulfoxide at $0.5 \%$. 

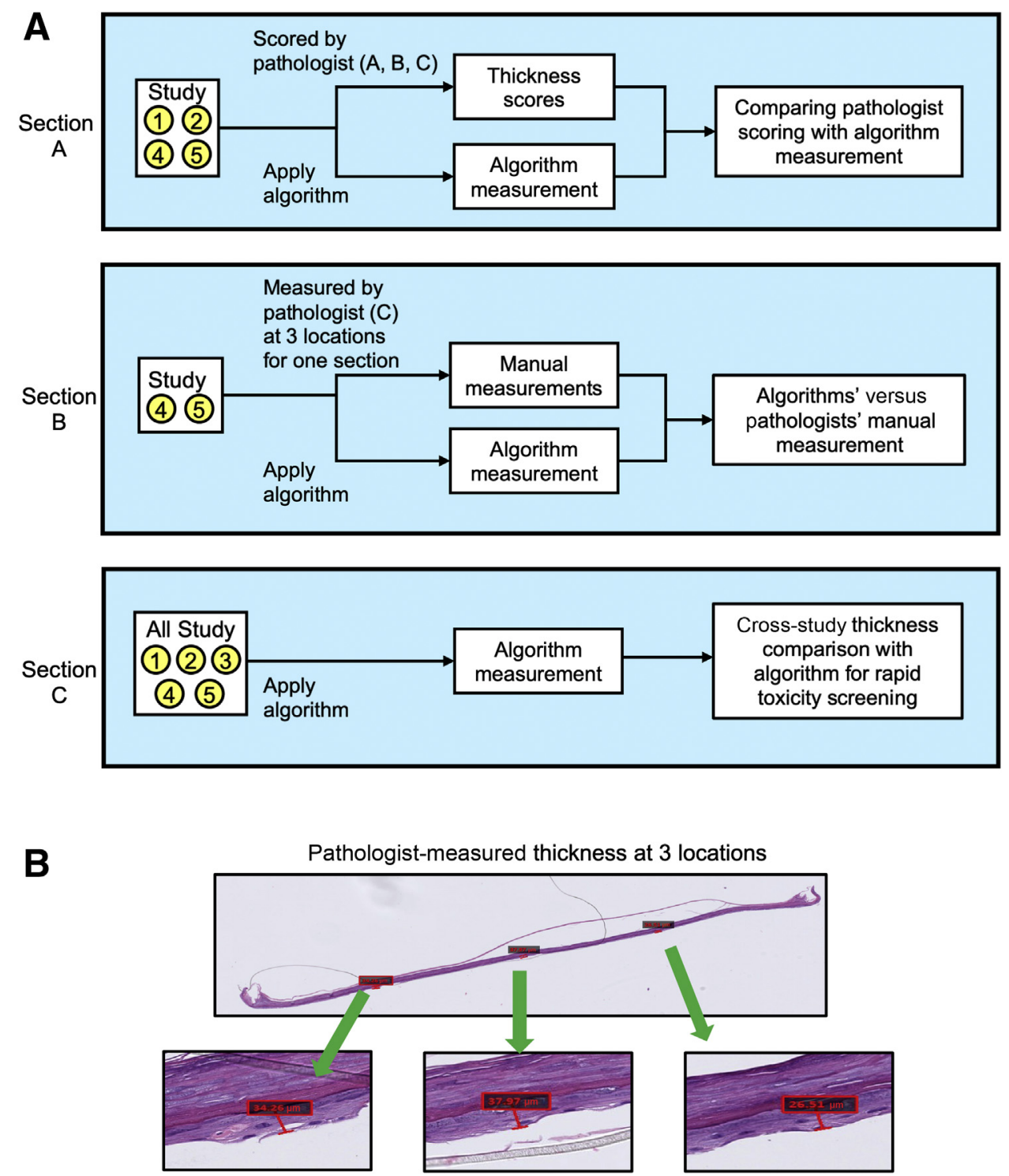

Figure 1 Study design. A: Sections A, B, and C summarize the outline of the article. The same trained algorithm was used to extract the viable epidermal thickness in all sections. Section A and Section B were used to understand the relationship between the manual end points and the algorithm-measured thickness. In Section A, three pathologists scored the thickness of the viable epidermis in the four studies, and the scores were compared against the algorithm-measured thickness. In Section B, a pathologist was provided with an image viewer to measure the viable epidermal thickness. The manual-measured thickness was also compared with the algorithm-measured thickness. In Section C, the algorithm-measured thickness for all samples was compared to enable rapid toxicity screening. B: Representative images from a pathologist measuring the viable epidermal thickness using an image viewer.

Supplemental Table S1 lists each compound, the study, and the different concentrations at which it was evaluated. Upon completion of treatment, the RHE samples were harvested, fixed, processed to slides, and routinely stained with hematoxylin and eosin. Internal test compounds with suspected skin toxicity were anonymized.

\section{Manual Annotation and Algorithm Training}

Pixel-level annotations of the keratin layer and the viable epidermal layer from nine RHE samples from Studies 1, 3, 4, and 5 (one sample from Study 1, four samples from Study 3, three samples from Study 4. and one sample from Study
5) were created by pathologist $C$ using Aperio ImageScope (Leica, Wetzlar, Germany) for the purpose of training. An additional nine RHE samples from all five studies (two samples from Studies 1, 2, 3, and 4, respectively, and one sample from Study 5) were selected and annotated by pathologist $\mathrm{C}$ for model testing. Image tiles with a width and height of 1024 pixels and the corresponding pixel-level annotations were extracted at $40 \times$ resolution for training the segmentation algorithm. A variation of the U-Net, ResNetUNet, was used for segmenting the keratin and the viable epidermal area. The encoder of the U-Net was replaced with a deep residual neural network (50-layer version), and the rest of the U-Net architecture remained the same. Model 


\begin{tabular}{|c|c|c|c|c|c|c|c|}
\hline Study & $\begin{array}{l}\text { No. of } \\
\text { compounds }\end{array}$ & $\begin{array}{l}\text { No. of } \\
\text { samples }\end{array}$ & $\begin{array}{l}\text { No. of control } \\
\text { samples }\end{array}$ & $\begin{array}{l}\text { No. of slides with pixel-level } \\
\text { annotation for training }\end{array}$ & $\begin{array}{l}\text { No. of slides with pixel-level } \\
\text { annotation for testing }\end{array}$ & $\begin{array}{l}\text { Pathologist } \\
\text { score? }\end{array}$ & $\begin{array}{l}\text { Manually } \\
\text { measured } \\
\text { thickness? }\end{array}$ \\
\hline 2 & 7 & 49 & 4 & 0 & 2 & Yes (B) & No \\
\hline 3 & 7 & 56 & 4 & 4 & 2 & No & No \\
\hline 4 & 6 & 64 & 4 & 3 & 2 & Yes (C) & Yes (C) \\
\hline
\end{tabular}

training was performed with a step size of 512 for 90 epochs with a batch size of eight using two NVIDIA P6000 graphics processing units (NVIDIA Corporation, Santa Clara, CA). Data augmentation techniques, ${ }^{27}$ including random rotation with interpolation (in the range of -45 to 45 degrees), cropping, scaling (in the range of 0.8 to 1.2 for both $x$ and $y$ axes independently), shifting, adding Gaussian noise (in the range of 0 to 12.75 per channel randomly), and varying color contrast, were applied during the training process. Focal loss $(\alpha=0.25$ and $\gamma=2)$ and Adam optimizer (learning rate $=1 \times 10^{-3}$ ) were used for optimization. The model performance for segmenting the viable epidermal area was evaluated by computing the dice coefficient of the manual-segmented and algorithm-predicted viable epidermal areas in the testing set.

\section{Image Processing and Model Inference}

For every RHE sample, the two RHE tissue sections were identified from the background glass using Otsu's method. For each RHE tissue section, the identified tissue area was cropped into image tiles with width and height of 1024 pixels. The trained ResNet-UNet was applied to the cropped image tiles and the output of the ResNet-UNet was resized into the shape of the input images. All RHE tissue tiles, identified with Otsu's method, were inferred by the ResNetUNet, and the keratin and epidermal areas were segmented. The algorithm outputs were examined by pathologists to ensure that both keratin and epidermal areas were captured.

After the epidermal area was identified, the thickness of the viable epidermal area was computed by using distance transformation. ${ }^{28,29}$ The median thickness value was used to represent the thickness of the viable epidermal area for each tissue section, and the average thickness of the two tissue sections of a RHE sample was used to represent a single RHE sample. Within each study, the thickness of each RHE sample was divided by the average thickness of the control for normalization.

\section{Manual Scoring and Measurement}

All RHE samples in Studies 1, 2, 4, and 5 were evaluated with optical microscopes by pathologists. The thickness of the viable epidermal area of the RHE samples in Studies 1 and 2 was scored by pathologists A and B, respectively, and
Studies 4 and 5 were scored by pathologist C. Each pathologist used a different scoring system to evaluate the thickness of the viable epidermal area. Pathologist A used the following system: marked epidermal thinning, moderate epidermal thinning, slight epidermal thinning, possible minimal decrease, no significant finding (same as control), and possible increase in epidermal thickness. Pathologist B used a scoring scale of 2 to 8 with the following ranges: 2 to 3 (thinnest), 3 to 4,4 to 5,6 to 7 , and 7 to 8 (same as control). Pathologist $\mathrm{C}$ used a scale of 0 to 4 , where 0 was the same as the control, increasing to 4 , the thinnest sample.

To enable cross-study comparison, the scores from pathologists were normalized. The scores for the control group were set to 1 and then readjusted so the higher the score, the thinner the viable epidermal thickness. Supplemental Table S2 summarizes the mapping from pathologist scores to the normalized scores.

In addition to the manual scoring, the viable epidermal thickness of each sample in studies 4 and 5 was measured with Aperio ImageScope (version 12.4.3) by pathologist C. Pathologist $\mathrm{C}$ chose three representative regions from each sample and measured the thickness of the RHE tissue (Figure 1B), which resulted in three measured epidermal thickness end points per sample. These three measurements were compared with the algorithm's measurements. These manual measurements also served to validate our algorithm's thickness measurement, providing pathologist insights regarding the closeness of the algorithm-measured thickness and the manual-measured thickness .

To aid in the interpretation of any meaningful change in epidermal thickness, the presence of specific microscopic characteristics was assessed. The evaluation was performed by all three pathologists and included a qualitative assessment of the stratum corneum and stratum granulosum, presence of parakeratosis, single or widespread keratinocyte necrosis, and evidence of keratinocyte swelling, usually due to vacuolation.

\section{Statistical Analyses}

Image analyses and statistical analyses were performed with Python version 3.6.7 (Python Software Corporation, Wilmington, DE). ResNet-UNet was trained using Keras (version 2.3.1)/TensorFlow (version 1.14.0, Google, Mountain View, CA). The training and inferring were 
Table 2 Summary of the Model Performance in the Training and Testing Sets

\begin{tabular}{|c|c|c|c|c|c|c|}
\hline Study & Data set & No. of samples & Treatment & Concentration, $\mu \mathrm{mol} / \mathrm{L}$ & Skin toxicity & Dice \\
\hline 1 & Training & 1 & DMSO & NA & No & 0.971 \\
\hline 3 & Training & 1 & DMSO & NA & No & 0.961 \\
\hline 3 & Training & 1 & G-0011 & 10 & Unknown & 0.975 \\
\hline 3 & Training & 1 & Cobimetinib & 1 & Yes & 0.932 \\
\hline 3 & Training & 1 & Pictilisib & 1 & Yes & 0.957 \\
\hline 4 & Training & 1 & DMSO & NA & No & 0.972 \\
\hline 5 & Training & 1 & Poziotinib & 0.3 & Yes & 0.970 \\
\hline 1 & Testing & 1 & Imatinib & 2 & Yes & 0.969 \\
\hline 1 & Testing & 1 & G-0001 & 0.5 & Unknown & 0.954 \\
\hline 2 & Testing & 1 & DMSO & NA & NA & 0.973 \\
\hline 2 & Testing & 1 & Alpelisib & 3.2 & Yes & 0.942 \\
\hline 5 & Testing & 1 & Osimertinib & 3 & Yes & 0.944 \\
\hline
\end{tabular}

The dice coefficients were computed from comparing the algorithm-generated masks versus the manually generated masks of the viable epidermal area only. The model achieved high dice coefficients in both training and testing sets.

DMSO, dimethyl sulfoxide; NA, not applicable.

performed by using graphics processing units (P6000, NVIDIA Corporation).

To evaluate the relationship between the pathologist scores and the algorithm-measured thickness, Kendall's Tau-b correlation coefficients were computed. The correlations were computed between the normalized pathologist scores and the normalized averaged thickness measured by the algorithm for Studies 1, 2, 4, and 5.

The quantitative thickness measurements in Studies 4 and 5 from the algorithm and pathologist $\mathrm{C}$ were also compared. It was expected that the difference between the two measurements would decrease as more manual measurements were made because the algorithm measured the thickness from the entire RHE, whereas the manual measurements were only made at three regions of interest (ROI). Therefore, a single thickness measurement was first randomly selected from the three measurements made from the pathologists. The randomly selected measurement for each sample was compared versus the average algorithm measurement for that sample. Next, for each sample, the average of the three measurements from pathologist $\mathrm{C}$ was compared with the average algorithm measurement. The Bland-Altman method was used to assess the agreement between the algorithmmeasured epidermal thickness and the thickness manually measured by pathologist $\mathrm{C}$ in Studies 4 and 5 .

To compare the rank orders across studies, the rank orders of the control samples were set to 1 . In addition, the rank order increased to a maximum of 5 with a decrease in viable epidermal thickness. Zero or negative rank orders would suggest an increase in viable epidermal thickness. The converted rank order is referred to as the normalized pathologist score for simplicity.
The mean thickness of the normalized viable epidermal thickness from all studies was grouped together and compared with the normalized viable epidermal thickness for each test compound. The same test compound and the same experimental protocol from different studies were combined. Tukey's multiple comparison method was also used.

\section{Results}

\section{Algorithm-Measured Viable Epidermal Thickness Significantly Correlates with Pathologist Scores}

Table 2 shows the computed dice coefficient, treatment, concentration, and skin toxicity of each sample in the training and testing sets. The model achieved an average dice coefficient of 0.953 and 0.956 for training and testing sets, respectively. Although the training data contained only nine samples (1083 independent tiles), the model achieved dice scores above 0.9 for all samples in the testing set, which included many unseen treatments and concentrations not present in the training set. Figure 2 displays representative results in case of the normalized epidermal thickness $>1.0$ or $<0.75$. Figure 3 shows boxplots comparing the algorithm-measured normalized epidermal thickness and the normalized scores from pathologist $\mathrm{A}$ in Study 1 (Figure 3A), pathologist B in Study 2 (Figure 3B), and pathologist $\mathrm{C}$ in Studies 4 and 5 (Figure 3, C and D, respectively). Significant correlations $(R=-0.62$ for Study $1,-0.76$ for Study 2, -0.67 for Study 4, and -0.84 for Study 5; all, $P<0.01$ ) were observed between the algorithm-measured viable epidermal thickness and the scores from pathologists. Notably, three samples in Study 1 


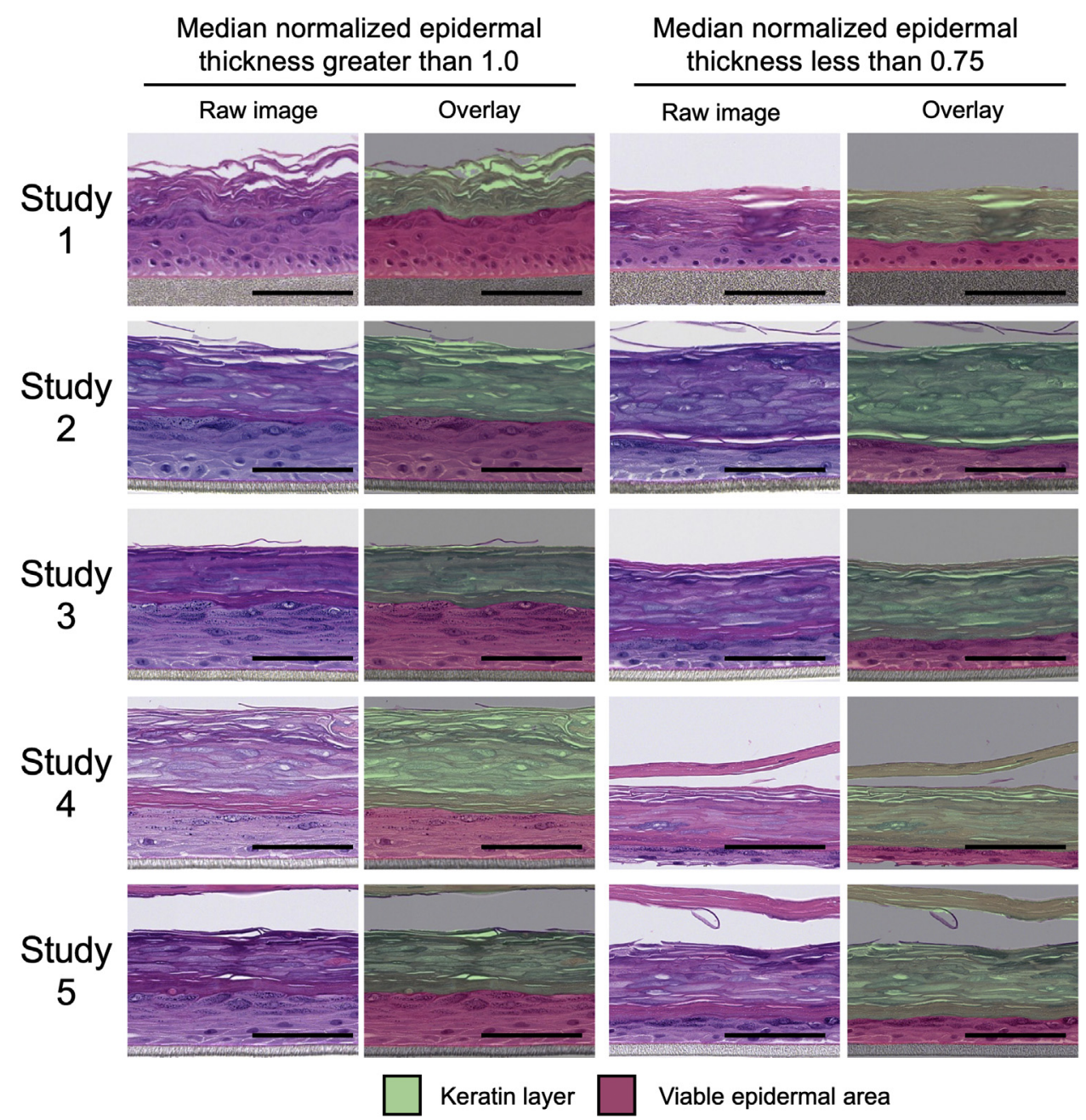

Figure 2 The trained deep learning algorithm can segment the viable epidermal area. Representative results for all studies from the trained deep learning algorithm are shown. The viable epidermal area and the keratin layer were accurately identified. Scale bars $=100 \mu \mathrm{m}$.

dosed at 10 times the clinically relevant concentration were diagnosed with full-thickness epidermal necrosis. The measured thickness for these three samples was zero (Figure 3A). In addition, in Study 4, due to microscopic finding of keratinocyte vacuolar degeneration with associated cell swelling in seven samples, the manual decrease in thickness score was lower than expected, which was reflected in the score calculated by the algorithm.

\section{Differences in Epidermal Thickness Decrease with Increasing Manual Measurements}

To provide more accurate comparisons between the pathologist thickness evaluation and the algorithm measurements of the epidermal area, pathologist $\mathrm{C}$ used Aperio ImageScope to manually measure the epidermal thickness of three ROIs from an RHE tissue. One manual measurement was randomly selected from the three ROIs and compared against the algorithm-measured thickness (Figure 4, A and B). The absolute errors between the algorithm-measured and the pathologist-measured epidermal thickness were $1.55 \pm 0.959 \mu \mathrm{m}$ and $1.25 \pm 1.200 \mu \mathrm{m}$ for Studies 4 and 5, respectively. When all three ROIs were used to compute the average measured thickness from the pathologist, the absolute errors between the algorithm-measured and the pathologist-measured epidermal thickness were reduced to $1.33 \pm 0.918 \mu \mathrm{m}$ and $1.02 \pm 0.943 \mu \mathrm{m}$ for Studies 4 and 5 , respectively (Figure 4, C and D). Overall, a good agreement between the algorithm and pathologists was achieved and was consistent over all thickness values. As the number of manual pathologist measurements and ROIs increased, the measured thickness became more similar to the algorithmmeasured results. Thus, multiple ROI led to smaller discrepancy and variance between manual measurements and the algorithm.

\section{Normalized Epidermal Thickness Enables Cross-Study Comparison and Reveals Potential Clinical Toxicity}

Data on normalized epidermal thickness of compounds with clinical observations were extracted from Studies 1, 2, 

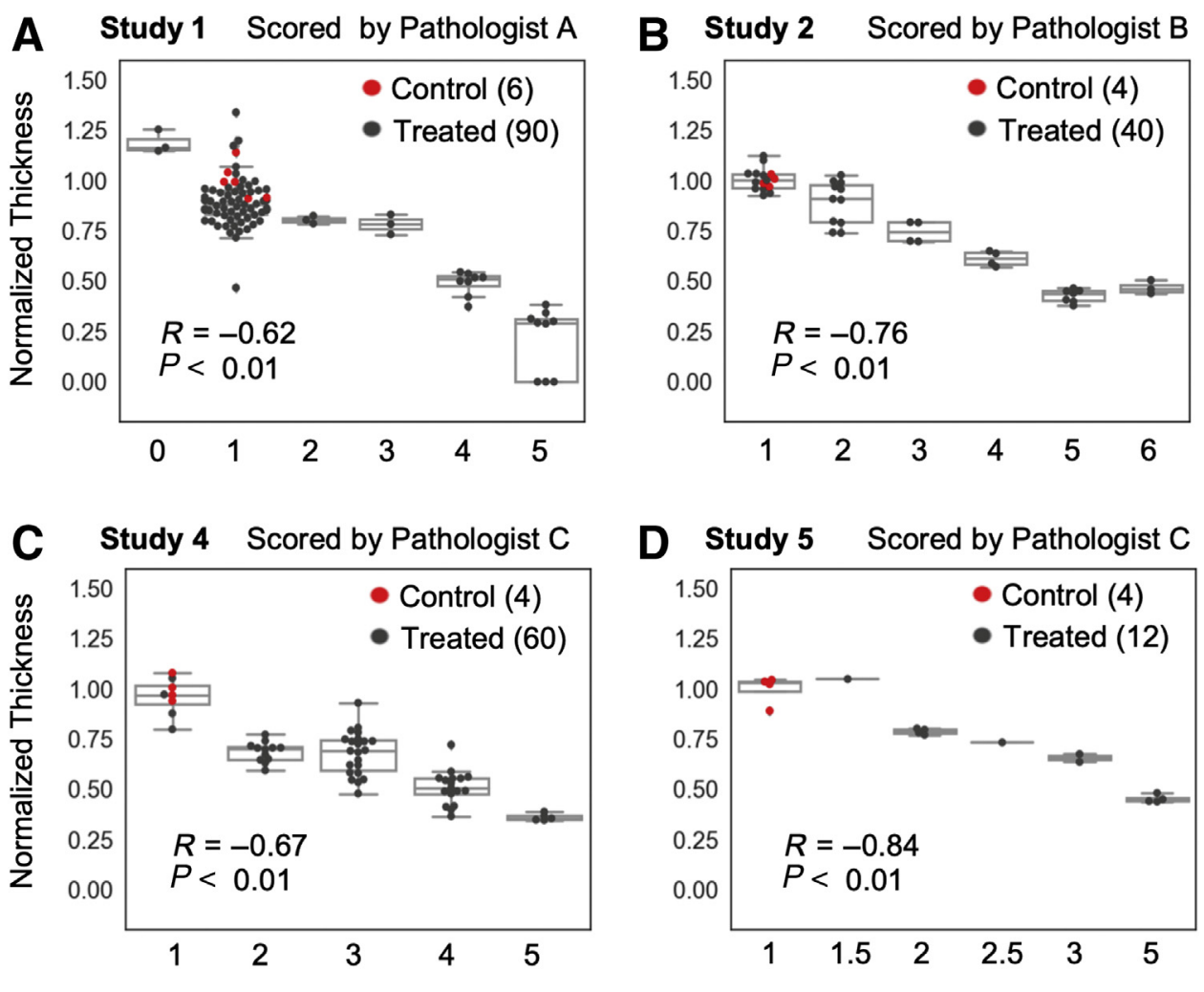

Normalized scores from pathologists (the higher the thinner)

Figure 3 Algorithm-measured epidermal thickness is significantly correlated with pathologist scoring. A-D: Boxplots comparing the normalized pathologist score and the algorithm-measured normalized thickness. The pathologist scores of the control group were set to 1 , and the scores were adjusted so that the higher the score, the thinner the viable epidermal thickness. The algorithm-measured epidermal thickness was normalized to the average thickness of the control group in each study. Pathologist scores were significantly correlated with the algorithm-measured thickness. Kendall's Tau-b correlation coefficients are shown.

3,4 , and 5 , and the correlation of epidermal thickness and clinical skin toxicity was analyzed retrospectively. Clinical skin toxicity was defined as rash, pruritus, acne, dermatitis, alopecia, and/or dry skin described in the labels for marketed compounds or in publications reporting safety of compounds under clinical development. Figure 5A shows the normalized viable epidermal thickness from various compounds under different concentrations. The results were ordered according to the median epidermal thickness from each experiment across all studies. Three samples in Study 1 that were dosed at 10 times the clinically relevant concentration were removed from this analysis. A total of eight compounds (afatinib, alpelisib, erlotinib, cobimetinib, osimertinib, poziotinib, lapatinib, and pictilisib) with a significant decrease in epidermal thickness exhibited clinical toxicity. Conversely, three compounds (tofacitinib, ruxolitinib, and Pf-04217903) with a significant decrease in epidermal thickness did not show clinical toxicity. Except for Pf-04217903 (10 $\mu \mathrm{mol} / \mathrm{L})$, all compounds with a normalized epidermal thickness decrease of $>20 \%$ exhibited clinical toxicity. Figure 5B shows the impact of the in vitro test concentration on the epidermal thickness for compounds exhibiting clinical skin toxicity and those that did not exhibit toxicity. Table 3 summarizes the test concentration and clinical plasma exposure to test compounds. ${ }^{30-46}$ Most of the skin-toxic compounds decreased epidermal thickness by $>20 \%$ at concentrations greater than fivefold of clinical exposure [maximal free concentrations measured in plasma $\left(\mathrm{C}_{\mathrm{max}-}\right.$ free $\left.)\right]$. In contrast, most of the nontoxic compounds decreased epidermal thickness by $<20 \%$ even at relatively high concentrations (fivefold higher than $\mathrm{C}_{\text {max }}$ free). Interestingly, a B-Raf inhibitor (XL-281) exhibited an increase in epidermal thickness both according to pathologist score and by the algorithm (albeit not statistically significant), indicating that compounds that induced hyperplastic toxicity can also be picked up with our algorithm. A cut of 0.80 (median normalized thickness from the osimertinib $300 \mathrm{nmol} / \mathrm{L}$ group) was used to compute the sensitivity and specificity for classifying skin toxicity with our algorithm. Table 4 summarizes the performance of using epidermal thickness alone and in combination with testing concentrations to identify skin-toxic compounds retrospectively. Given the fact that only one compound in this retrospective analysis causes hyperplastic toxicity, it is impossible to identify a threshold for an increase of epidermal thickness to capture skin toxicity. Therefore, XL-281 was not included in performance analysis. 

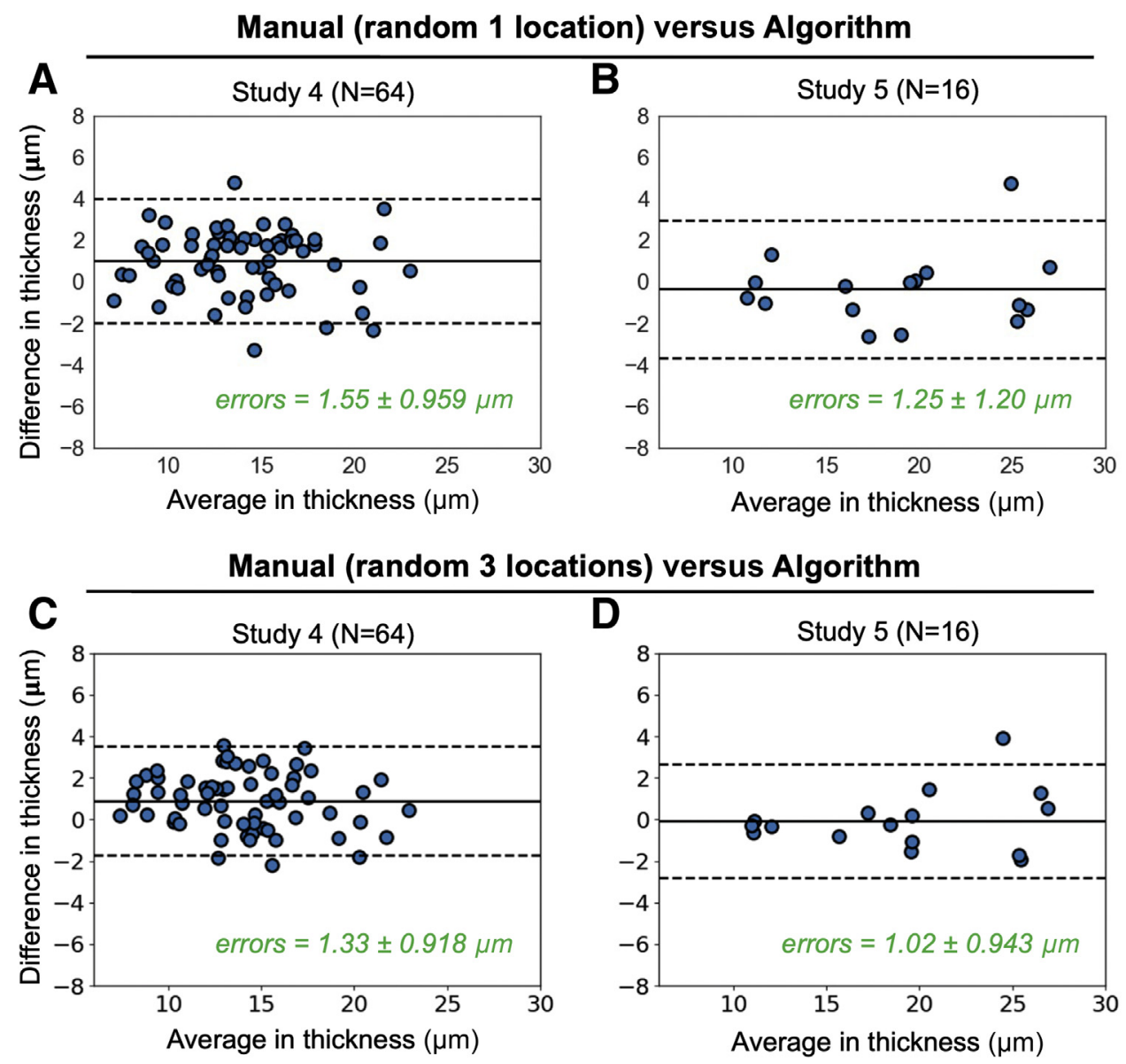

Figure 4 Manually measured epidermal thickness at more locations resulted in smaller errors. Bland-Altman plots comparing pathologist-measured viable epidermal thickness with algorithm-measured viable epidermal thickness. The averaged measured thicknesses from multiple locations ( $x$ axis) versus the algorithm-measured viable epidermal thickness ( $y$ axis) is shown. Pathologist $C$ measured the viable epidermal thickness at one region of interest from a single tissue section for every sample in Study 4 (A) and Study 5 (B). The absolute error and the SD were $1.55 \pm 0.959 \mu \mathrm{m}$ and $1.25 \pm 1.20 \mu \mathrm{m}$ for Study 4 and Study 5, respectively. Pathologist $C$ measured the viable epidermal thickness at three regions of interest from a single tissue section for every sample in Study 4 (C) and Study 5 (D). The absolute error and the SD were $1.33 \pm 0.918 \mu \mathrm{m}$ and $1.02 \pm 0.943 \mu \mathrm{m}$ for Study 4 and Study 5 , respectively. Agreement between the segmentation algorithm and pathologists was consistent over all thickness values. The dashed lines denote an approximate $95 \%$ CI for the residuals between the segmentation model and the manually measured thickness.

\section{Discussion}

The current study is the first to identify a $20 \%$ reduction of epidermal thickness as the threshold to differentiate technical variations (can happen to nontoxic compounds) and a real risk signal. The $20 \%$ epidermal thickness reduction is difficult to score manually. In fact, many of the skin-toxic compounds tested caused only 20\% to $30 \%$ epidermal thickness reduction even at high concentrations [>30-fold clinical exposure (eg, afatinib, cobimetinib, poziotinib, osimertinib, sorafenib)]. This underscores the need for a quantitative and objective scoring method. Although histopathology represents an important source of descriptive data in biomedical research, the use of tissue scoring has enhanced reproducibility of research studies. However, this traditional method of pathologic scoring is semi-quantitative and thus is subjective to bias and can be very timeconsuming. As shown by the data collected, by using algorithm-measured thickness, the scoring could be better understood and further normalized. The deep learning-based image analysis used in the current study empowered a rapid, quantitative, and accurate measurement of epidermal thickness to capture subtle reductions, on the micron level, that would normally go unnoticed with traditional pathologic evaluation.

Lack of labeled data is a common digital pathology due to the highly specialized nature of the pathology domain and time-consuming nature of the annotation process. This poses a challenge, as limited labeled data for training could potentially result in overfitting. ${ }^{47,48}$ Therefore, data augmentation is particularly important and often applied in a digital pathology workflow to improve model generalizability. ${ }^{27}$ Many standard data augmentation techniques were used in the current study. For example, mild random scaling was applied to account for slight variation in size and shape of histologic features. ${ }^{27}$ To explore generalizability, an aggressive 50/50 


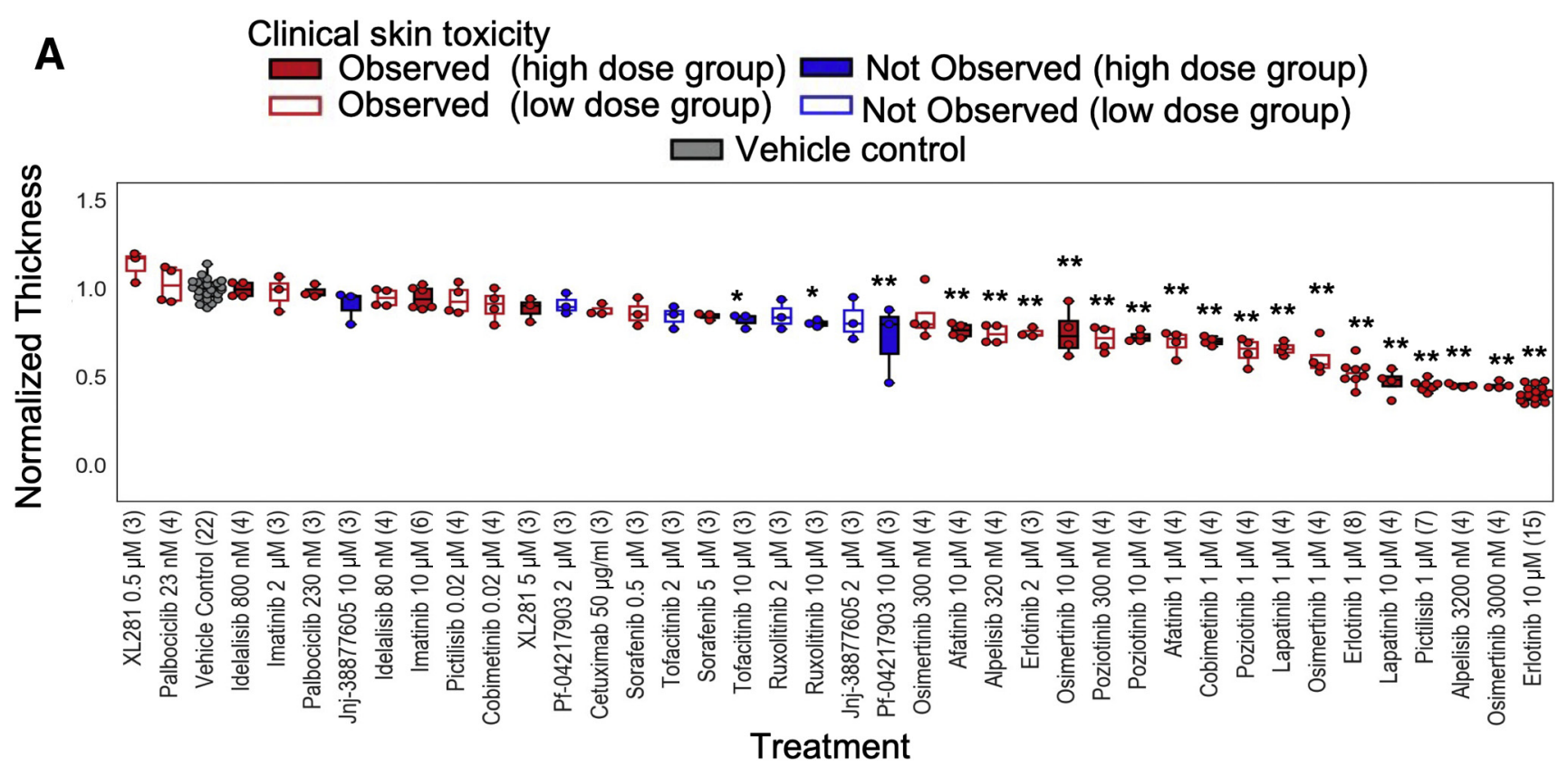

B

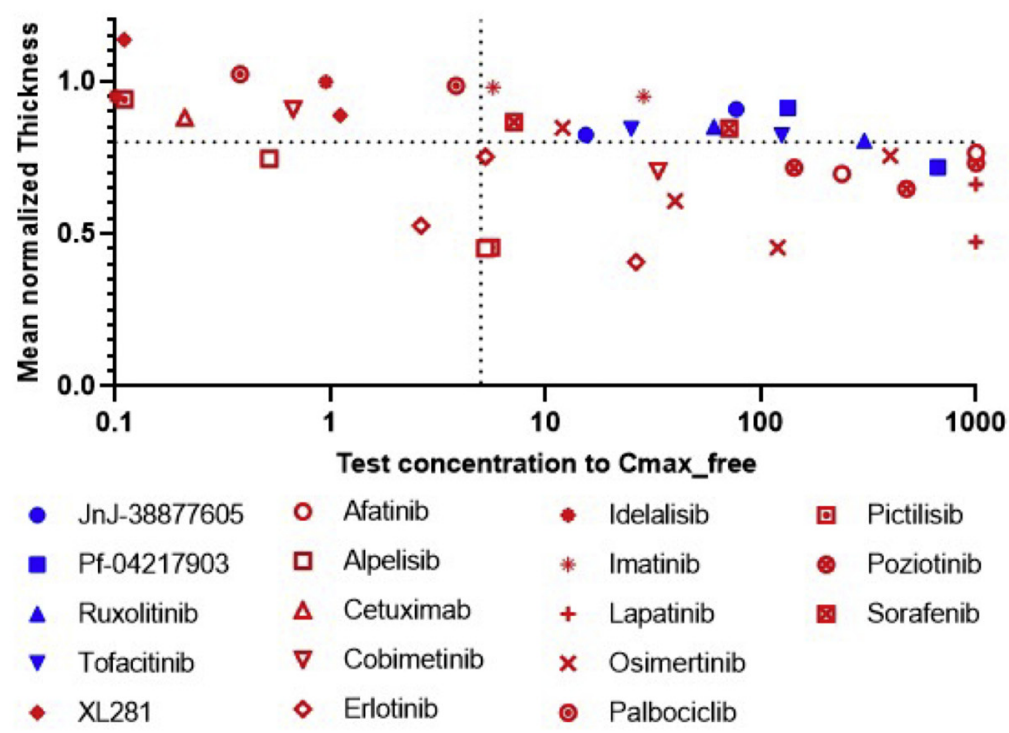

Figure 5 Changes in viable epidermal thickness might suggest clinical toxicity. A: Algorithm-computed normalized thickness for different compounds. The boxes are sorted according to the median value of the normalized thickness. Tukey's multiple comparison method was used. Numbers in the parentheses show the sample size. B: Mean value of normalized thickness for different compounds in the context of test concentrations and clinical exposure. Red dots represent skin-toxic compounds. Blue dots represent nontoxic compounds. Horizontal dashed line indicates $20 \%$ decrease in normalized thickness. Vertical dashed line indicates fivefold increase in concentration compared with the clinical exposure. ${ }^{*} P<0.05,{ }^{* *} P<0.01$. $C_{\max }$ free, maximal free concentrations measured in plasma.

data splitting strategy (nine training and nine testing samples) was used. Moreover, the testing set contains unseen sample conditions (the combination of study, treatments, concentrations, and whether drugs induce skin toxicity) not present in the training set to rule out potential confounds. Table 2 showed that the model achieved dice coefficients $>0.93$ for all testing samples. Furthermore, when applying the model to samples without corresponding annotations, segmented viable epidermal areas were visually checked for correctness and approved by pathologists. This suggested that the model was generalized well, and confounds might be minimal in the current study. It is likely that the features extracted by the model were similar in the training and testing sets so that the model achieved a good generalizability with augmented training data. Future direction includes expanding the training and testing data sets to further improve model robustness. Therefore, new features introduced in the latest studies are more likely to be identified and the viable epidermal areas are more likely to be segmented by the new model trained with the larger data set. 
Table 3 Comparison of Test Concentration and Clinical Plasma Exposure to Test Compounds

\begin{tabular}{|c|c|c|c|c|c|c|}
\hline Compound & $\begin{array}{l}\text { Concentration, } \\
\mu \mathrm{mol} / \mathrm{L}\end{array}$ & $C_{\max }$ free, $\mu \mathrm{mol} / \mathrm{L}$ & PK reference & Fold to $C_{\max }$ free & $\begin{array}{l}\text { Skin } \\
\text { toxicity }\end{array}$ & $\begin{array}{l}\text { Normalized } \\
\text { thickness }\end{array}$ \\
\hline Jnj-38877605 & 10 & $0.13^{*}$ & 30,31 & 76.9 & No & 0.91 \\
\hline Jnj-38877605 & 2 & $0.13^{*}$ & 30,31 & 15.4 & No & 0.82 \\
\hline Pf-04217903 & 10 & 0.015 & 32 & 666.7 & No & 0.72 \\
\hline Pf-04217903 & 2 & 0.015 & 32 & 133.3 & No & 0.91 \\
\hline Ruxolitinib & 10 & 0.033 & 33,34 & 303.0 & No & 0.81 \\
\hline Tofacitinib & 10 & 0.08 & tofacitinib label; 35 & 125.0 & No & 0.82 \\
\hline XL281 & 0.5 & $4.5^{*}$ & 36 & 0.1 & No & 1.14 \\
\hline XL281 & 5 & $4.5^{*}$ & 36 & 1.1 & No & 0.89 \\
\hline Afatinib & 10 & 0.0042 & 37 & $>1000$ & Yes & 0.77 \\
\hline Afatinib & 1 & 0.0042 & 37 & 238.1 & Yes & 0.70 \\
\hline Alpelisib & 0.32 & 0.61 & alpelisib label & 0.5 & Yes & 0.75 \\
\hline Cobimetinib & 1 & 0.03 & cobimetinib label & 33.3 & Yes & 0.71 \\
\hline Erlotinib & 2 & 0.38 & 38 & 5.3 & Yes & 0.75 \\
\hline Erlotinib & 1 & 0.38 & 38 & 2.6 & Yes & 0.53 \\
\hline Erlotinib & 10 & 0.38 & 38 & 26.3 & Yes & 0.41 \\
\hline Idelalisib & 0.8 & 0.84 & idelalisib label; 39 & 1.0 & Yes & 1.00 \\
\hline Idelalisib & 0.08 & 0.84 & idelalisib label; 39 & 0.1 & Yes & 0.95 \\
\hline Imatinib & 10 & 0.35 & 40 & 28.6 & Yes & 0.95 \\
\hline Imatinib & 2 & 0.35 & 40 & 5.7 & Yes & 0.98 \\
\hline Lapatinib & 1 & 0.000039 & lapatinib label & $>1000$ & Yes & 0.66 \\
\hline Lapatinib & 10 & 0.000039 & lapatinib label & $>1000$ & Yes & 0.47 \\
\hline Osimertinib & 0.3 & 0.025 & 41 & 12.0 & Yes & 0.85 \\
\hline Poziotinib & 0.3 & 0.0021 & 45 & 142.9 & Yes & 0.72 \\
\hline Poziotinib & 1 & 0.0021 & 45 & 476.2 & Yes & 0.65 \\
\hline Sorafenib & 0.5 & 0.07 & 46 & 7.1 & Yes & 0.87 \\
\hline Sorafenib & 5 & 0.07 & 46 & 71.4 & Yes & 0.85 \\
\hline
\end{tabular}

Information on US Food and Drug Administration-approved drugs can be found at https://www.accessdata.fda.gov/scripts/cder/daf/index.cfm. *Plasma protein binding unavailable. Total concentration used in fold calculation.

$\mathrm{C}_{\text {max }}$ free, maximal free concentrations measured in plasma; $\mathrm{PK}$, pharmacokinetics.

The lack of quantitative end points makes it difficult to evaluate thickness, particularly for studies with large sample sizes. Significant correlations were observed between the algorithm-measured epidermal thickness and pathologist scores for Studies 1, 2, 4, and 5. In addition, the smaller the sample size, the stronger the correlation $(R=-0.84$, $-0.76,-0.67$, and -0.62 when $N=16,44,64$, and 96, respectively) (Figure 3 ). This finding suggested that manual evaluation of the epidermal thickness might be more challenging as sample size increases. Epidermal thickness varied from location to location within an RHE; while more consistent thickness is present in the central portion of the tissue, harvesting-related artifacts at the edge of the samples and detachment of the basal lamina further challenge the scoring process. To capture any meaningful variation, the pathologists typically selected multiple ROI in adequately preserved areas, took measurements, and reported the median value from the selected regions. ${ }^{49}$ Figure 4 shows that digital tools minimized the discrepancy between manualand algorithm-based measurements to $<2 \mu \mathrm{m}$. Furthermore, this discrepancy was reduced as more manual measurements were made.

Several factors can potentially affect the manual measurements made by pathologists. For example, different 
Assess Skin Toxicity with Deep Learning

Table 4 Performance Summary of Epidermal Thickness Alone and in Combination with Testing Concentration to Identify Skin-Toxic Compounds Retrospectively

\begin{tabular}{|c|c|c|c|c|c|c|c|}
\hline Inclusion criteria & $\begin{array}{l}\text { No. of } \\
\text { drugs }\end{array}$ & $\begin{array}{l}\text { True } \\
\text { positive }\end{array}$ & $\begin{array}{l}\text { False } \\
\text { positive }\end{array}$ & True negative & $\begin{array}{l}\text { False } \\
\text { negative }\end{array}$ & Sensitivity & Specificity \\
\hline All compounds (excluding XL-281) & 17 & 8 & 1 & 3 & 5 & 0.62 & 0.8 \\
\hline
\end{tabular}

manual methods could result in varying thickness measurements. ${ }^{50}$ Thickness measured vertically from the basement membrane could easily vary from measurements made perpendicular to the basement membrane for the same sample. The number of measured regions could also affect the measured value due to thickness variation of the epidermal area. Lastly, ROI selection and the measurement starting and ending point could be difficult to reproduce. It is therefore possible that the same sample could result in different thickness values during repetitive experiments. Although the results showed that the discrepancy between manual and algorithm measurements was small, the algorithm may provide an advantage when changes are subtle and manual measurements are not recommended, particularly when a large sample size was involved.

One major caveat of the EpiDerm model is that it does not contain melanocytes or an immune component. Human skin is characterized by a variable number of epidermal and hair melanocytes, and it contains various immune cell populations, including Langerhans cells, macrophages, mast cells, and $\mathrm{T}$ cells in the dermis. The lack of the aforementioned components renders the EpiDerm model of limited immunologic significance and precludes the evaluation of melanocyte contribution to drug-induced cutaneous toxicity. Nevertheless, the sole presence of keratinocytes may provide the advantage of examining the drug effects on a single-cell population without any confounding influences from other cell populations. ${ }^{14}$ Aside from measuring the epidermal thickness, the qualitative microscopic evaluation of the samples provides further insights into pathologic findings that may influence the thickness itself. For example, the presence of widespread epidermal necrosis with no remaining measurable layer translated into a score of zero. Conversely, the presence of vacuolar degeneration with enlarged, swollen keratinocytes translated into a minor decrease in epidermal thickness, thus leading to a score that was lower than expected. In addition, the effect of drugs on differentiation of keratinocytes such as an increased or decreased granular layer cannot be quantified and still needs to be evaluated by a pathologist. As such, a qualitative microscopic evaluation remains pivotal in uncovering underlying factors that may influence the interpretation of the automated scoring system for epidermal thickness.

In the oncology field, a papulopustular rash mostly affecting face and upper trunk has been reported in a high percentage of patients treated with EGFR inhibitors. ${ }^{7}$ This on-target, off-tumor effect is not surprising and, given that this toxicity represents an indicator of clinical efficacy, patients are often dosed to rash. Our retrospective analysis confirmed that the EpiDerm model is a useful tool for measuring skin toxicity after EGFR inhibition (afatinib, erlotinib, lapatinib, osimertinib, and poziotinib as examples). In addition, the EpiDerm model is responsive to drugs that inhibit phosphoinositide 3-kinase $\alpha / \delta$ (pictilisib), phosphoinositide 3-kinase $\alpha$ (alpelisib), and mitogenactivated protein kinase kinase (cobimetinib), recapitulating the clinical cutaneous toxicity phenotype. ${ }^{8,14,51}$ In comparison, the kinase inhibitors imatinib (up to 29-fold over clinical $\mathrm{C}_{\mathrm{max}}$ free) and sorafenib (up to 71-fold over clinical $\mathrm{C}_{\mathrm{max}}$ free) failed to cause any meaningful change in epidermal thickness (Figure 5A). Because immune mechanisms seem to be involved in the imatinib-associated skin rash observed clinically, ${ }^{52}$ it is not surprising that epidermal effects are not reproduced by the RHE model. Similarly, for sorafenib, the higher drug concentration observed in the areas of rash appears to be related to the eccrine activity of the skin, which cannot be recapitulated by the RHE model given the absence of sweat glands. ${ }^{53}$ As such, the EpiDerm model may not be sensitive to certain signal pathway inhibitor-induced skin toxicity, including BCR-ABL and Raf, with few caveats. Adverse epidermal proliferative lesions have been reported in patients with BRAFV600-mutated metastatic melanoma when treated with certain B-Raf inhibitors due to paradoxical mitogenactivated protein kinase pathway activation. ${ }^{8,54}$ In this instance, the epidermal hyperplasia induced by these B-Raf inhibitors at lower pharmacologically relevant concentrations appears to be reproduced by the EpiDerm model, while manifesting as epidermal thinning and toxicity at suprapharmacologic higher concentrations. ${ }^{14}$ Here, increased epidermal thickness has been confirmed in the Raf inhibitor-treated EpiDerm model and correlated with epidermal hyperplasia and the induction of epidermal tumors observed preclinically ${ }^{55}$ and clinically. ${ }^{56}$ Thus, the predictive value of the EpiDerm model is dependent on the mechanism of action as well as the concentration of each compound tested.

Given the limited assessment of compounds causing epidermal thickening, the current study failed to identify a threshold for this finding. However, compounds that enhance cytokines such as IL-22 and oncostatin M, as well as compounds that inhibit the phosphorylation of serine/threonine 
protein kinase and its downstream target, mammalian target of rapamycin, have shown the ability to induce morphologic and differentiation features in RHE that closely mimic epidermal psoriasiform changes in patients. ${ }^{14,57}$

The prediction value of the EpiDerm model should also be put into context of compound testing concentrations. The current study is also the first report to identify that a minimum testing concentration of fivefold anticipated clinical exposure is necessary to improve sensitivity and to avoid false-negative results. Nontoxic compounds cause $<20 \%$ epidermal thinning regardless of the testing concentration (from 0.1-fold to 300-fold over clinical $\mathrm{C}_{\text {max }}$ free). Thus, the specificity of the EpiDerm model is similar across different inclusion criteria for the testing concentrations. However, the retrospective analysis showed that skin-toxic compounds decreased epidermal thickness when tested at concentrations equivalent to fivefold the therapeutic clinical exposure (Figure 5B). The sensitivity of the EpiDerm model increased from 0.62 to 0.8 , and the specificity decreased from 0.8 to 0.75 when adding the testing concentration in the inclusion criteria for predictively analysis (Table 4). Skin toxicity typically develops acutely within 1 or 2 weeks after treatment, although peaks can be observed after 2 to 4 weeks or more. ${ }^{9,58,59}$ In the current study, the EpiDerm model was treated with test compounds for 4 days, which is shorter than the time course for skin toxicity manifestation in the clinic. Due to these considerations, the compound concentrations required to cause changes in the epidermal thickening in the EpiDerm model may be higher than the maximal exposure seen in the clinic. Thus, the EpiDerm model may only have good predictive value when compounds are tested at concentrations equivalent to fivefold the therapeutic clinical exposure or higher.

Deep learning and image processing algorithms can provide quantitative end points of epidermal thickness. The current study showed that this computed epidermal thickness from the EpiDerm model could reflect potential clinical skin toxicity. This approach is fully automated, scalable, and reproducible. Therefore, it can potentially enhance the throughput for compound screening. With time, as more quantitative data are collected, confidence in predicting clinical skin toxicity will only grow.

\section{Acknowledgments}

We thank Dr. Gopinath Palanisamy for his evaluation of the skin samples, Ms. Susan Haller for processing the samples and preparing the slides, and Dr. Ruth Sullivan and Dr. Reina Fuji for providing feedback for this research.

\section{Supplemental Data}

Supplemental material for this article can be found at http://doi.org/10.1016/j.ajpath.2021.12.007.

\section{References}

1. Tran QT, Kennedy LH, Leon Carrion S, Bodreddigari S, Goodwin SB, Sutter CH, Sutter TR: EGFR regulation of epidermal barrier function. Physiol Genomics 2012, 44:455-469

2. Hou D-D, Di Z-H, Qi R-Q, Wang H-X, Zheng S, Hong Y-X, Guo H, Chen H-D, Gao X-H: Sea Buckthorn (Hippophaë rhamnoides L.) oil improves atopic dermatitis-like skin lesions via inhibition of NF-[kappa] B and STAT1 activation. Skin Pharmacol Physiol 2017, 30:268-276

3. Rizzo HL, Kagami S, Phillips KG, Kurtz SE, Jacques SL, Blauvelt A: IL-23-mediated psoriasis-like epidermal hyperplasia is dependent on IL-17A. J Immunol 2011, 186:1495-1502

4. Jost M, Kari C, Rodeck U: The EGF receptor-an essential regulator of multiple epidermal functions. Eur J Dermatol 2000, 10:505-510

5. Pastore S, Mascia F, Mariani V, Girolomoni G: The epidermal growth factor receptor system in skin repair and inflammation. J Invest Dermatol 2008, 128:1365-1374

6. Schneider MR, Werner S, Paus R, Wolf E: Beyond wavy hairs: the epidermal growth factor receptor and its ligands in skin biology and pathology. Am J Pathol 2008, 173:14-24

7. Lacouture ME: Mechanisms of cutaneous toxicities to EGFR inhibitors. Nat Rev Cancer 2006, 6:803-812

8. Macdonald JB, Macdonald B, Golitz LE, LoRusso P, Sekulic A: Cutaneous adverse effects of targeted therapies: part II: inhibitors of intracellular molecular signaling pathways. J Am Acad Dermatol 2015, 72:221-236. quiz 237-238

9. Nunnery SE, Mayer IA: Management of toxicity to isoform alphaspecific PI3K inhibitors. Ann Oncol 2019, 30:x21-x26

10. Hardwick RN, Betts CJ, Whritenour J, Sura R, Thamsen M, Kaufman EH, Fabre K: Drug-induced skin toxicity: gaps in preclinical testing cascade as opportunities for complex in vitro models and assays. Lab Chip 2020, 20:199-214

11. Faller C, Bracher M, Dami N, Roguet R: Predictive ability of reconstructed human epidermis equivalents for the assessment of skin irritation of cosmetics. Toxicol In Vitro 2002, 16:557-572

12. Kandárová H, Liebsch M, Gerner I, Schmidt E, Genschow E, Traue D, Spielmann H: The EpiDerm test protocol for the upcoming ECVAM validation study on in vitro skin irritation tests-an assessment of the performance of the optimised test. Altern Lab Anim 2005, 33:351-367

13. Kandárová H, Liebsch M, Spielmann H, Genschow E, Schmidt E, Traue D, Guest R, Whittingham A, Warren N, Gamer AO, Remmele M, Kaufmann T, Wittmer E, De Wever B, Rosdy M: Assessment of the human epidermis model SkinEthic RHE for in vitro skin corrosion testing of chemicals according to new OECD TG 431. Toxicol In Vitro 2006, 20:547-559

14. Danilenko DM, Phillips GDL, Diaz D: In vitro skin models and their predictability in defining normal and disease biology, pharmacology, and toxicity. Toxicol Pathol 2016, 44:555-563

15. Ekins S: The next era: deep learning in pharmaceutical research. Pharm Res 2016, 33:2594-2603

16. LeCun Y, Bengio Y, Hinton G: Deep learning. Nature 2015, 521 : 436-444

17. Hilton CB, Milinovich A, Felix C, Vakharia N, Crone T, Donovan C, Proctor A, Nazha A: Personalized predictions of patient outcomes during and after hospitalization using artificial intelligence. NPJ Digit Med 2020, 3:51

18. Senior AW, Evans R, Jumper J, Kirkpatrick J, Sifre L, Green T, Qin C, Žídek A, Nelson AWR, Bridgland A, Penedones H, Petersen S, Simonyan K, Crossan S, Kohli P, Jones DT, Silver D, Kavukcuoglu K, Hassabis D: Improved protein structure prediction using potentials from deep learning. Nature 2020, 577:706-710

19. Zuraw A, Staup M, Klopfleisch R, Aeffner F, Brown D, WesterlingBui T, Rudmann D: Developing a qualification and verification strategy for digital tissue image analysis in toxicological pathology. Toxicol Pathol 2021, 49:773-783 
20. Jimenez-Carretero D, Abrishami V, Fernández-de-Manuel L, Palacios I, Quíez-Álvarez A, Díez-Sánchez A, Del Pozo MA, Montoya MC: Tox_(R)CNN: deep learning-based nuclei profiling tool for drug toxicity screening. PLoS Comput Biol 2018, 14:e1006238

21. Maddah M, Mandegar MA, Dame K, Grafton F, Loewke K, Ribeiro AJS: Quantifying drug-induced structural toxicity in hepatocytes and cardiomyocytes derived from hiPSCs using a deep learning method. J Pharmacol Toxicol Methods 2020, 105:106895

22. De Vera Mudry MC, Martin J, Schumacher V, Venugopal R: Deep learning in toxicologic pathology: a new approach to evaluate rodent retinal atrophy. Toxicol Pathol 2021, 49:851-861

23. Hu F, Schutt L, Kozlowski C, Regan K, Dybdal N, Schutten MM: Ovarian toxicity assessment in histopathological images using deep learning. Toxicol Pathol 2020, 48:350-361

24. Tokarz DA, Steinbach TJ, Lokhande A, Srivastava G, Ugalmugle R, Co CA, Shockley KR, Singletary E, Cesta MF, Thomas HC, Chen VS, Hobbie K, Crabbs TA: Using artificial intelligence to detect, classify, and objectively score severity of rodent cardiomyopathy. Toxicol Pathol 2021, 49:888-896

25. Ibragimov B, Toesca D, Chang D, Yuan Y, Koong A, Xing L: Development of deep neural network for individualized hepatobiliary toxicity prediction after liver SBRT. Med Phys 2018, 45:4763-4774

26. Srivastava A, Hanig JP: Quantitative neurotoxicology: potential role of artificial intelligence/deep learning approach. J Appl Toxicol 2021, 41:996-1006

27. Berman AG, Orchard WR, Gehrung M, Markowetz F: PathML: a unified framework for whole-slide image analysis with deep learning. medRxiv 2021, [Preprint] doi: 10.1101/2021.07.07.21260138

28. Hu F, Martin H, Martinez A, Everitt J, Erkanli A, Lee WT, Dewhirst M, Ramanujam N: Distinct angiogenic changes during carcinogenesis defined by novel label-free dark-field imaging in a hamster cheek pouch model. Cancer Res 2017, 77:7109-7119

29. Hu F, Morhard R, Murphy HA, Zhu C, Ramanujam N: Dark field optical imaging reveals vascular changes in an inducible hamster cheek pouch model during carcinogenesis. Biomed Opt Express 2016, 7:3247-3261

30. Caillat-Zucman S, Garchon HJ, Timsit J, Assan R, Boitard C, DjilaliSaiah I, Bougnères P, Bach JF: Age-dependent HLA genetic heterogeneity of type 1 insulin-dependent diabetes mellitus. J Clin Invest 1992, 90:2242-2250

31. Lolkema MP, Bohets HH, Arkenau H-T, Lampo A, Barale E, de Jonge MJA, van Doorn L, Hellemans P, de Bono JS, Eskens FALM: The c-Met tyrosine kinase inhibitor JNJ-38877605 causes renal toxicity through species-specific insoluble metabolite formation. Clin Cancer Res 2015, 21:2297-2304

32. Yamazaki S, Skaptason J, Romero D, Vekich S, Jones HM, Tan W, Wilner KD, Koudriakova T: Prediction of oral pharmacokinetics of cMet kinase inhibitors in humans: physiologically based pharmacokinetic model versus traditional one-compartment model. Drug Metab Dispos 2011, 39:383-393

33. De Marinis E, Ceccherelli A, Quattrocchi A, Leboffe L, Polticelli F, Nervi C, Ascenzi P: Ruxolitinib binding to human serum albumin: bioinformatics, biochemical and functional characterization in JAK2V617F ${ }^{+}$cell models. Sci Rep 2019, 9:16379

34. Shilling AD, Nedza FM, Emm T, Diamond S, McKeever E, Punwani N, Williams W, Arvanitis A, Galya LG, Li M, Shepard S, Rodgers J, Yue TY, Yeleswaram S: Metabolism, excretion, and pharmacokinetics of [14C] INCB018424, a selective Janus tyrosine kinase $1 / 2$ inhibitor, in humans. Drug Metab Dispos 2010, 38:2023-2031

35. Cada DJ, Demaris K, Levien TL, Baker DE: Tofacitinib. Hosp Pharm 2013, 48:413-424

36. Dickson MA, Gordon MS, Edelman G, Bendell JC, Kudchadkar RR, LoRusso PM, Johnston SH, Clary DO, Schwartz GK: Phase I study of XL281 (BMS-908662), a potent oral RAF kinase inhibitor, in patients with advanced solid tumors. Invest New Drugs 2015, 33: $349-356$
37. Wind S, Schnell D, Ebner T, Freiwald M, Stopfer P: Clinical pharmacokinetics and pharmacodynamics of afatinib. Clin Pharmacokinet 2017, 56:235-250

38. Hidalgo M, Bloedow D: Pharmacokinetics and pharmacodynamics: maximizing the clinical potential of erlotinib (Tarceva). Semin Oncol 2003, 30(Suppl 7):25-33

39. Piper WL, Waddell JA, Solimando DA Jr: Drug monographs: belinostat and idelalisib. Hosp Pharm 2014, 49:1009-1013

40. Peng B, Hayes M, Resta D, Racine-Poon A, Druker BJ, Talpaz M, Sawyers CL, Rosamilia M, Ford J, Lloyd P, Capdeville R: Pharmacokinetics and pharmacodynamics of imatinib in a phase I trial with chronic myeloid leukemia patients. J Clin Oncol 2004, 22:935-942

41. Grande E, Harvey RD, You B, Batlle JF, Galbraith H, Sarantopoulos J, Ramalingam SS, Mann H, So K, Johnson M, Vishwanathan K: Pharmacokinetic study of osimertinib in cancer patients with mild or moderate hepatic impairment. J Pharmacol Exp Ther 2019, 369:291-299

42. Tamura K, Mukai H, Naito Y, Yonemori K, Kodaira M, Tanabe Y, Yamamoto N, Osera S, Sasaki M, Mori Y, Hashigaki S, Nagasawa T, Umeyama Y, Yoshino T: Phase I study of palbociclib, a cyclindependent kinase 4/6 inhibitor, in Japanese patients. Cancer Sci 2016, 107:755-763

43. Salphati L, Pang J, Plise EG, Chou B, Halladay JS, Olivero AG, Rudewicz PJ, Tian Q, Wong S, Zhang X: Preclinical pharmacokinetics of the novel PI3K inhibitor GDC-0941 and prediction of its pharmacokinetics and efficacy in human. Xenobiotica 2011, 41:1088-1099

44. Sarker D, Ang JE, Baird R, Kristeleit R, Shah K, Moreno V, Clarke PA, Raynaud FI, Levy G, Ware JA, Mazina K, Lin R, Wu J, Fredrickson J, Spoerke JM, Lackner MR, Yan Y, Friedman LS, Kaye SB, Derynck MK, Workman P, de Bono JS: First-in-human phase I study of pictilisib (GDC-0941), a potent pan-class I phosphatidylinositol-3-kinase (PI3K) inhibitor, in patients with advanced solid tumors. Clin Cancer Res 2015, 21:77-86

45. Noh Y-H, Lim H-S, Jung J-A, Song TH, Bae K-S: Population pharmacokinetics of HM781-36 (poziotinib), pan-human EGF receptor (HER) inhibitor, and its two metabolites in patients with advanced solid malignancies. Cancer Chemother Pharmacol 2015, 75:97-109

46. Minami H, Kawada K, Ebi H, Kitagawa K, Kim Y-I, Araki K, Mukai H, Tahara M, Nakajima H, Nakajima K: Phase I and pharmacokinetic study of sorafenib, an oral multikinase inhibitor, in Japanese patients with advanced refractory solid tumors. Cancer Sci 2008, 99:1492-1498

47. Shorten C, Khoshgoftaar TM: A survey on image data augmentation for deep learning. J Big Data 2019, 6:60

48. Tizhoosh HR, Pantanowitz L: Artificial intelligence and digital pathology: challenges and opportunities. J Pathol Inform 2018, 9:38

49. Gibson-Corley KN, Olivier AK, Meyerholz DK: Principles for valic histopathologic scoring in research. Vet Pathol 2013, 50:1007-1015

50. Turin SY, Ledwon JK, Bae H, Buganza-Tepole A, Topczewska J, Gosain AK: Digital analysis yields more reliable and accurate measures of dermal and epidermal thickness in histologically processed specimens compared to traditional methods. Exp Dermatol 2018, 27: 687-690

51. Hanker AB, Kaklamani V, Arteaga CL: Challenges for the clinical development of PI3K inhibitors: strategies to improve their impact in solid tumors. Cancer Discov 2019, 9:482-491

52. Park SR, Ryu M-H, Ryoo B-Y, Beck MY, Lee IS, Choi MJ, Lee MW, Kang Y-K: Severe imatinib-associated skin rash in gastrointestinal stromal tumor patients: management and clinical implications. Cancer Res Treat 2016, 48:162-170

53. Lai SE, Kuzel T, Lacouture ME: Hand-foot and stump syndrome to sorafenib. J Clin Oncol 2007, 25:341-343

54. Mandalà M, Massi D, De Giorgi V: Cutaneous toxicities of BRAF inhibitors: clinical and pathological challenges and call to action. Crit Rev Oncol Hematol 2013, 88:318-337 
55. Doma E, Rupp C, Varga A, Kern F, Riegler B, Baccarini M: Skin tumorigenesis stimulated by Raf inhibitors relies upon Raf functions that are dependent and independent of ERK. Cancer Res 2013, 73: 6926-6937

56. Schwartz GK, Robertson S, Shen A, Wang E, Pace L, Dials H, Mendelson D, Shannon P, Gordon M: A phase I study of XL281, a selective oral RAF kinase inhibitor, in patients (Pts) with advanced solid tumors. J Clin Oncol 2009, 27:3513

57. Datta Mitra A, Raychaudhuri SP, Abria CJ, Mitra A, Wright R, Ray R, Kundu-Raychaudhuri S: 1[alpha],25-Dihydroxyvitamin-D3- 3-bromoacetate regulates AKT/mTOR signaling cascades: a therapeutic agent for psoriasis. J Invest Dermatol 2013, 133:1556-1564

58. Manousaridis I, Mavridou S, Goerdt S, Leverkus M, Utikal J: Cutaneous side effects of inhibitors of the RAS/RAF/MEK/ERK signalling pathway and their management. J Eur Acad Dermatol Venereol 2013, 27:11-18

59. Scope A, Agero ALC, Dusza SW, Myskowski PL, Lieb JA, Saltz L, Kemeny NE, Halpern AC: Randomized double-blind trial of prophylactic oral minocycline and topical tazarotene for cetuximabassociated acne-like eruption. J Clin Oncol 2007, 25:5390-5396 